\title{
Stellar Wobble Due to a Nearby Binary System
}

\author{
M. H. M. Morais ${ }^{1}$ and A. C. M. Correia ${ }^{2}$ \\ ${ }^{1}$ Department of Physics, I3N, University of Aveiro, \\ Campus Universitario de Santiago, 3810-193 Aveiro, Portugal \\ email: helena.morais@ua.pt \\ 2 Astronomie et Systemes Dynamiques, IMCCE-CNRS UMR 8028, \\ 77 Avenue Denfert-Rochereau, 75014 Paris, France \\ email: correia@ua.pt
}

\begin{abstract}
To date, there are several reported exoplanet detections within binary star systems. These findings are based on radial velocity data for the target star. However, the companion star could in turn have a companion of which we are not aware. We describe how this hidden binary system affects the radial velocity of the target star, mimicking a planet in some circumstances We also explain what can be done in practice in order to distinguish between these two effects.
\end{abstract}

Keywords. (stars:) binaries: general,(stars:) planetary systems, techniques: radial velocities

\section{Overview of theory and results}

We study a triple system composed of a star with mass $M_{\star}$, at distance $\vec{r}$ from the centre of mass of a binary with masses $M_{1}+M_{2}$, and inter-binary distance $\vec{r}_{b}$. We assume that $\left|\overrightarrow{r_{b}}\right| /|\vec{r}| \ll 1$ (hierarchical system) thus the motion is, approximately, a composition of two Keplerian orbits described by: $\vec{r}_{b}$, with semi-major axis $a_{b}$, eccentricity $e_{b}$, and period $T_{b}=2 \pi / n_{b}$; and $\vec{r}$, with semi-major axis $a$, eccentricity $e$, and period $T=2 \pi / n$.

The star's radial velocity is $V_{R}=V_{R K}+V_{R P}$, where $V_{R K}$ is a Keplerian term that describes the motion around a "star", of mass $M_{1}+M_{2}$, located at the binary system's centre of mass, and $V_{R P}$ is a small perturbation[1,2]. Therefore, the radial velocity data of a star with a nearby unresolved binary system is first fitted with a Keplerian curve, $V_{R K}$. After subtracting $V_{R K}$, we are left with the perturbation term, $V_{R P}$, from which we could, in principle, infer the presence of the hidden binary component. However, as we will show next, this is not always possible in practice.

Coplanar circular orbits:

In the case of coplanar circular orbits we have [1]

$$
V_{R P}=K_{0} \cos \left(n t+\lambda_{0}\right)+K_{1} \cos \left(\left(2 n_{b}-3 n\right) t+\lambda_{1}\right)+K_{2} \cos \left(\left(2 n_{b}-n\right) t+\lambda_{2}\right) .
$$

The term with frequency $n$ is incorporated in the main Keplerian curve, $V_{R K}$. Since $n_{b} \gg n$, the 2nd and 3rd terms have very close frequencies that can only be resolved if the observation timespan $t_{o b s} \geqslant T / 2$. If there is enough resolution and precision, we identify both signals and we conclude that they should not be caused by planets (as such close orbits would be unstable). However, since $\left|K_{1}\right|=5\left|K_{2}\right|$, in practice, due to limited precision, we may only be able to observe the signal with frequency $2 n_{b}-3 n$. In this case, we may think there is a planet companion to the observed star.

We simulated a coplanar triple system composed of $M_{\star}=M_{\odot}$ on a circular orbit of period $T=22 \mathrm{y}$, around binary $M_{1}=0.7 M_{\odot}$ and $M_{2}=0.35 M_{\odot}$ with circular orbit of period $T_{b}=411 \mathrm{~d}$. We computed radial velocity data points over $t_{o b s}=11 \mathrm{y}$ at precision 


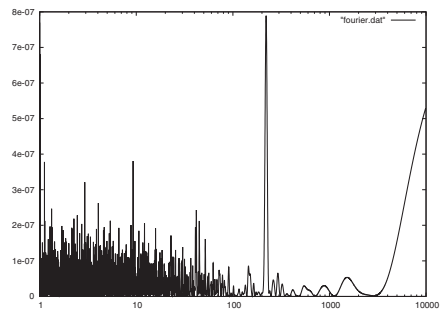

(a)

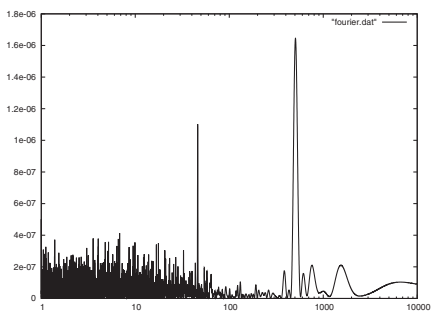

(b)

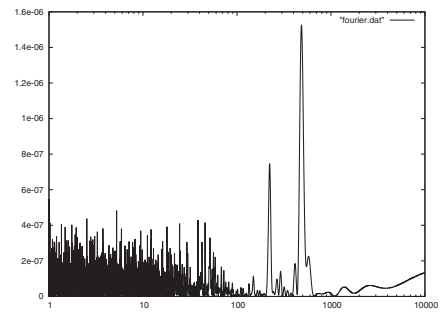

(c)

Figure 1: Fourier spectrum of residuals leftover after removing $V_{R K}$.

$0.8 \mathrm{~m} / \mathrm{s}$. In Fig. 1(a) we see a signal with frequency $2 n_{b}-3 n$ (amplitude $0.9 \mathrm{~m} / \mathrm{s}$ at $223 \mathrm{~d}$ ) that mimics a planet of $18 M_{E}$.

Non-coplanar circular orbits:

In the case of non-coplanar circular orbits, $V_{R P}$ is a combination of 6 periodic terms[2] with frequencies: $n, 3 n, 2 n_{b} \pm n$ and $2 n_{b} \pm 3 n$. Depending on the observation precision and resolution we may observe one or more of these terms. If all these have well separated frequencies we may mistake them for planet(s).

We simulated a triple system composed of $M_{\star}=M_{\odot}$ on a circular orbit of period $T=$ $4.2 \mathrm{y}$, around binary $M_{1}=M_{2}=0.25 M_{\odot}$ with circular orbit of period $T_{b}=85 \mathrm{~d}$. The relative inclination is $i=30^{\circ}$. We computed radial velocity data points over $t_{o b s}=11 \mathrm{y}$ at precision $0.7 \mathrm{~m} / \mathrm{s}$. In Fig. 1(b) we see signals with frequency $2 n_{b}-3 n$ (amplitude $0.8 \mathrm{~m} / \mathrm{s}$ at $46 \mathrm{~d}$ ), and frequency $3 n$ (amplitude $1.4 \mathrm{~m} / \mathrm{s}$ at $516 \mathrm{~d}$ ) that mimic planets of $7 M_{E}$ and $20 M_{E}$.

Eccentric coplanar orbits:

It can be shown that, generally, $V_{R P}$ is a composition of periodic terms [2]. In the eccentric $2 \mathrm{D}$ case, up to 1 st order in the eccentricities, there are 12 frequencies: $n, 2 n_{b}-n$, $2 n_{b}-3 n, n_{b}-n, n_{b}-3 n, 3 n_{b}-n, 3 n_{b}-3 n, n_{b}+n, 2 n, 2 n_{b}, 2 n_{b}-4 n$ and $2 n_{b}-2 n$.

We simulated a coplanar triple system composed of $M_{\star}=M_{\odot}$ with $e=0.1$ and period $T=22 \mathrm{y}$, around binary $M_{1}=0.7 M_{\odot}$ and $M_{2}=0.35 M_{\odot}$ with $e_{b}=0.2$ and period $T_{b}=411 \mathrm{~d}$. We computed radial velocity data points over $t_{o b s}=11 \mathrm{y}$ at precision $0.8 \mathrm{~m} / \mathrm{s}$. In Fig. 1(c) we see signals with frequencies $2 n_{b}-3 n$ (amplitude $0.8 \mathrm{~m} / \mathrm{s}$ at $223 \mathrm{~d}$ ) and $n_{b}-3 n$ (amplitude $1.4 \mathrm{~m} / \mathrm{s}$ at $487 \mathrm{~d}$ ). Since $n \ll n_{b}$ these mimic planets of $15 M_{E}$ and $34 M_{E}$ at the $2: 1$ mean motion resonance.

If $t_{o b s} \gg T$, signals at or nearby harmonics of $n$ appear [3]. These may be mistaken by planet(s) in mean motion resonance with a companion "star" of mass $M_{1}+M_{2}$. As $t_{\text {obs }}$ increases, the short period terms described here become negligible with respect to the orbits' secular evolution [3].

Distinguishing planet from binary:

We showed that, in order to avoid erroneous announcements of new planets, we need to have precise observations over a reasonably long timespan, which is often not possible in practice. However, a signal with frequency, $n_{p l}$, and amplitude, $K_{p l}$, mimics a planet with parameters [1,2] $a_{p l}=\left(G M_{\star}\right)^{1 / 3} / n_{p l}^{2 / 3}$ and $M_{p l} \sin I_{p l}=K_{p l}\left(M_{\star}+M_{1}+M_{2}\right) /\left(n_{p l} a_{p l}\right)$. Therefore, we can invert these expressions to predict the binary system parameters that can mimic a given planet and check if they are realistic $[1,2]$.

\section{References}

[1 ] Morais, M. H. M. \& Correia, A. C. M. 2008, A\& $A$ A, 391, 899

[2 ] Morais, M. H. M. \& Correia, A. C. M. 2011, $A \& A A, 525, \mathrm{~A} 152$

[3 ] Morais, M. H. M. \& Correia, A. C. M. 2011, MNRAS, submitted 